Ciência Florestal, Santa Maria, v. 23, n. 3, p. 411-416, jul.-set., 2013

ISSN 0103-9954

\title{
SOBREVIVÊNCIA E CRESCIMENTO DE MUDAS DE REGENERAÇ̃̃O NATURAL DE Astronium gracile Engl. EM CLAREIRAS CAUSADAS POR EXPLORAÇÃO FLORESTAL NA AMAZÔNIA BRASILEIRA
}

\author{
SURVIVAL AND GROWTH RATE OF SEEDLINGS FROM NATURAL REGENERATION OF \\ Astronium gracile Engl. IN GAPS CAUSED BY REDUCED IMPACT LOGGING IN THE BRAZILIAN \\ AMAZON
}

Larissa Corrêa Lopes Quadros ${ }^{1}$ João Olegário Pereira de Carvalho² Jaqueline Macêdo Gomes ${ }^{3}$ Marisol Taffarel ${ }^{4}$ Joice Carolina Fernandes Silva ${ }^{5}$

\section{RESUMO}

Avaliou-se a sobrevivência e o crescimento em altura de indivíduos da regeneração natural de Astronium gracile Engl., em diferentes tamanhos de clareiras ocasionadas pela exploração florestal no município de Paragominas, Pará. As mudas de Astronium gracile foram selecionadas aleatoriamente nas clareiras. As medições foram feitas nos anos de 2005, 2006, 2008 e 2010. As clareiras foram classificadas em pequenas $\left(200-400 \mathrm{~m}^{2}\right)$, médias $\left(401-600 \mathrm{~m}^{2}\right)$ e grandes $\left(>600 \mathrm{~m}^{2}\right)$. As mudas de regeneração natural de Astronium gracile, espécie considerada tolerante à sombra, se beneficiaram da abertura de clareiras, principalmente das maiores, para crescer em altura e para sobreviver, demonstrando potencial para uso em sistemas silviculturais, visando aumentar o valor comercial da floresta para futuras colheitas de madeira.

Palavras-chave: floresta de terra firme; tratamento silvicultural; manejo florestal.

\begin{abstract}
The survival and the height growth rates of individuals from the natural regeneration of Astronium gracile Engl. were evaluated in different gap sizes after logging in the municipality of Paragominas, Pará state. Seedlings of Astronium gracile were randomly chosen in the gaps. The measurements were performed in 2005, 2006, 2008 and 2010. The canopy gaps were classified as small gaps (200-400 $\left.\mathrm{m}^{2}\right)$, medium gaps $\left(401-600 \mathrm{~m}^{2}\right)$ and large gaps $\left(>600 \mathrm{~m}^{2}\right)$. The seedlings from natural regeneration of Astronium gracile, considered as a shade-tolerant species, were favored by the open gaps, mainly large gaps, for growing up and surviving, showing potential for using in silvicultural systems to increase the commercial value of the forest and to allow future harvests.
\end{abstract}

Keywords: terra firme forest; silvicultural treatments; natural forest management.

1 Engenheira Florestal, Doutoranda do Programa de Pós-Graduação em Ciências Florestais, Universidade Federal Rural da Amazônia, Av. Presidente Tancredo Neves, 2501, Bairro Montese, CEP 66077-901, Belém (PA). larissa.quadros@ufra.edu.br

2 Engenheiro Florestal, D.Phil., Professor Visitante Nacional Sênior (CAPES/CNPq/UFRA), Av. Presidente Tancredo Neves, 2501, Bairro Montese, CEP 66077-901, Belém (PA). olegario@pq.cnpq.br

3 Engenheira Florestal, Doutoranda do Programa de Pós-Graduação em Ciências Florestais, Universidade Federal Rural da Amazônia, Av. Presidente Tancredo Neves, 2501, Bairro Montese, CEP 66077-901, Belém (PA). jaquelinemacedogomes@hotmail.com

4 Engenheira Florestal, Mestre em Ciências Florestais, Universidade Federal Rural da Amazônia, Av. Presidente Tancredo Neves, 2501, Bairro Montese, CEP 66077-901, Belém (PA). marisoltaffarel@hotmail.com

5 Engenheira Florestal, Mestre do Programa de Pós-Graduação em Ciências Florestais, Universidade Federal Rural da Amazônia, Av. Presidente Tancredo Neves, 2501, Bairro Montese, CEP 66077-901, Belém (PA).joice eng.florestal@yahoo.com.br

Recebido para publicação em 17/06/2011 e aceito em 11/05/2012 


\section{INTRODUÇÃO}

Estudos sobre a dinâmica de espécies arbóreas comerciais são de extrema importância para dar suporte ao manejo florestal, pois informações básicas, como crescimento em altura e diâmetro, ingresso, sobrevivência e mortalidade, associadas à formação de clareiras, são importantes para preconizar os melhores tratamentos silviculturais para beneficiar determinadas espécies.

Os tratamentos silviculturais são técnicas utilizadas pelo silvicultor para promover o melhor desempenho de espécies comerciais e agregar mais valor à floresta. Segundo Gomes et al. (2010), os principais tratamentos realizados em florestas tropicais são: corte de cipós, liberação de copas para maior captação de luz, condução da regeneração natural e enriquecimento de clareiras.

Um grande número de espécies florestais depende da abertura de clareiras para a sua regeneração (HARTSHORN, 1980). A formação de clareiras no dossel florestal gera alterações ambientais na floresta e a colonização dessas clareiras por espécies de diferentes categorias sucessionais, presentes no banco de plântulas ou de sementes do solo e/ ou de indivíduos remanescentes (MARTINS et al., 2008).

Segundo Bazzaz e Pickett (1980), pequenas clareiras favorecem o crescimento de regeneração avançada, como é o caso de plântulas ou mudas já estabelecidas antes da formação da clareira. Orians (1982) afirma que muitas espécies comuns a grandes áreas alteradas são raras em simples clareiras, sugerindo que o tamanho da clareira pode ser uma importante variável que afeta a germinação de sementes e a sobrevivência de plântulas.

Denslow (1980) dividiu as espécies em três grupos de acordo com a sua demanda por luz: espécies especialistas em grandes clareiras, que necessitam de condições de alta luminosidade para germinar; espécies especialistas em pequenas clareiras, que germinam na sombra formando banco de plântulas e que necessitam de clareiras para crescerem até o dossel; e espécies especialistas de sub-bosque, que não necessitam de clareiras para germinar nem para atingir a maturidade reprodutiva.

Caron et al. (2010) afirmam que a diversidade de resposta à luminosidade é grande, sobretudo quanto ao crescimento e ao desenvolvimento vegetativo da parte aérea e à sobrevivência das mudas. Para Scalon e Alvarenga (1993), a eficiência do crescimento das plantas pode ser relacionada com a habilidade de adaptação das plântulas às condições luminosas do ambiente.

A ausência da regeneração natural de espécies comerciais depois da colheita de madeira e a elevada competição dos indivíduos remanescentes com espécies sem valor de mercado são algumas das causas que põem em risco a sustentabilidade do manejo de florestas tropicais (OLIVEIRA et al., 2006). O plantio de enriquecimento em clareiras é uma alternativa viável para promover e aumentar a abundância de espécies de valor comercial, entretanto, para utilizar esse tratamento silvicultural é necessário saber como essas espécies se comportam em relação às clareiras.

Diversos estudos têm sido realizados sobre o comportamento de espécies florestais em exposição à radiação solar (PINTO et al., 1993; RONDON NETO et al., 2000; SANSEVERO et al., 2006; JARDIM et al., 2007; JARDIM e SOARES, 2010). Estudar o comportamento de espécies arbóreas em diferentes intensidades de luz é necessário para conhecer o seu potencial silvicultural e garantir a sustentabilidade de espécies que têm dificuldades na sua regeneração.

Nesse sentido, no presente estudo, avaliou-se a sobrevivência e o crescimento em altura de indivíduos da regeneração natural de Astronium gracile Engl., em diferentes tamanhos de clareiras ocasionadas pela exploração florestal de impacto reduzido no município de Paragominas-Pará.

\section{MATERIAL E MÉTODO}

\section{Área de estudo}

O estudo foi realizado na Fazenda Rio Capim, pertencente à empresa Cikel Brasil Verde Madeiras Ltda., localizada no município de Paragominas, PA, entre as coordenadas $2^{\circ} 25^{\prime}$ e $4^{\circ} 09^{\prime} \mathrm{S}$ e $46^{\circ} 25^{\prime}$ e $48^{\circ} 54^{\prime} \mathrm{W}$. O estudo de plantio em clareiras faz parte do Projeto "Silvicultura pós-colheita na Amazônia Brasileira - Projeto SPC", instalado pela Embrapa Amazônia Oriental em $2005 \mathrm{em} 700$ ha de floresta. A área do experimento foi explorada em 2004.

Segundo a classificação de Köppen, o clima predominante na região é do tipo "Aw", isto é, tropical chuvoso com estação seca bem definida, caracterizado por temperatura média anual de $27,2^{\circ} \mathrm{C}$, com umidade relativa do ar de $81 \%$ e precipitação pluviométrica com média de $1.766 \mathrm{~mm} / \mathrm{ano}$, com ocorrência de menos disponibilidade hídrica no 
período de julho a outubro (WATRIN e ROCHA, 1992).

A topografia da área vai de plana a suavemente ondulada. As principais classes de solos, identificadas na região, de acordo com Rodrigues et al. (2003), são: Latossolos Amarelos, Argissolos Amarelos, Plintossolos, Gleissolos e Neossolos. Os solos possuem baixa fertilidade, devido à baixa reserva de nutrientes como cálcio, magnésio, potássio, fósforo e nitrogênio, além de alta saturação por alumínio.

A tipologia da área onde foram instalados os tratamentos silviculturais é Floresta Ombrófila Densa, também chamada de Floresta Equatorial Úmida de Terra Firme (VELOSO et al., 1991).

\section{Amostragem e obtenção dos dados}

Os dados do Projeto SPC, no qual a presente pesquisa está inserida, foram coletados em 400 clareiras causadas por queda de árvores colhidas durante a exploração de impacto reduzido realizada na área. As clareiras foram selecionadas em 200 ha de floresta explorada, portanto em média duas clareiras por hectare, com tamanhos variando de $200 \mathrm{~m}^{2}$ a $1230 \mathrm{~m}^{2}$. As clareiras foram separadas convencionalmente em três classes de tamanho: pequenas $\left(200-400 \mathrm{~m}^{2}\right)$; médias $\left(401-600 \mathrm{~m}^{2}\right)$; e grandes (área $>600 \mathrm{~m}^{2}$ ). Com base em informações da literatura sobre o crescimento em altura e em diâmetro e na lista de espécies exploradas pela empresa Cikel, foram selecionadas mudas de regeneração natural de 51 espécies para serem monitoradas nas clareiras. Houve também o plantio de mudas de 17 espécies nas mesmas clareiras, mas isso não é discutido no presente estudo.

A espécie Astronium gracile Engl., selecionada para esta pesquisa, está presente em 97 clareiras e possui 167 indivíduos. As mudas foram selecionadas aleatoriamente nas clareiras, obedecendo a uma distância de aproximadamente $5 \mathrm{~m}$ entre si, ou entre mudas das outras espécies também selecionadas da regeneração natural ou mudas plantadas. As avaliações das mudas foram realizadas em quatro ocasiões: 2005, 2006, 2008 e 2010, quando se registrou a presença (sobrevivência) ou ausência (morte) e mediu-se a altura de cada indivíduo.

\section{Análise dos dados}

Realizaram-se as análises da sobrevivência e crescimento em altura da espécie. Para a sobre- vivência, foram contabilizados os indivíduos que sobreviveram entre as medições. $\mathrm{O}$ crescimento foi calculado através do Incremento Periódico Anual em altura (IPAh), por meio da expressão:

$$
\mathrm{IPA}_{\mathrm{h}}=\frac{\text { Medição }_{\text {final }}-\text { Medição }_{\text {inicial }}}{\mathrm{Tempo}_{\text {final }}-\mathrm{Tempo}_{\text {inicial }}}
$$

Os resultados obtidos foram submetidos à análise de variância e as médias foram comparadas pelo teste de Tukey a 5\% de probabilidade.

\section{RESULTADOS E DISCUSSÃO}

\section{Sobrevivência}

A maior taxa de sobrevivência após a última avaliação (2010) foi registrada nas clareiras grandes $(95,60 \%)$, seguida das clareiras pequenas $(90,11 \%)$ e médias $(89,01 \%)$ (Figura 1$)$. Esses valores indicam que essa espécie, mesmo sendo considerada por Carvalho (2000) como tolerante à sombra, seguindo a classificação de Swaine e Whitmore (1988), beneficia-se da abertura de clareiras.

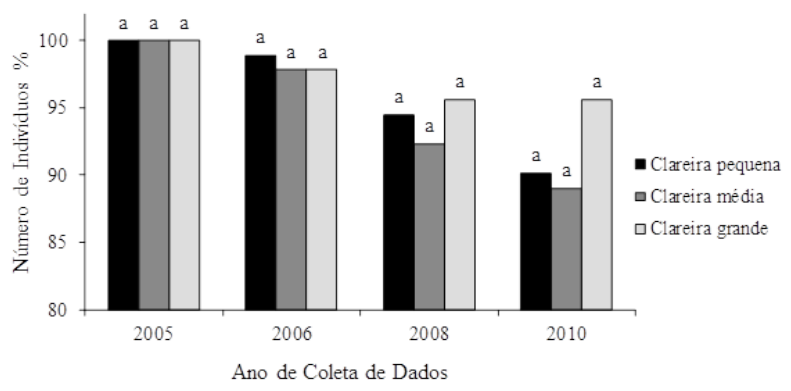

FIGURA 1: Sobrevivência de mudas de regeneração natural de Astronium gracile Engl., no período de cinco anos, em diferentes tamanhos de clareiras em uma área de floresta natural de terra firme na fazenda Rio Capim, município de Paragominas, PA. Médias seguidas pela mesma letra não apresentam diferenças estatísticas entre si.

FIGURE 1: Survival of seedlings from natural regeneration of Astronium gracile Engl. in a five-year period in different gap sizes in a terra firme natural forest in the Rio Capim Forest Management Unit, municipality of Paragominas, Pará state. Bars with the same letter are not significantly different at $p<0,05$. 
A sobrevivência das mudas da espécie foi alta durante todo o período estudado (Figura 1). Nas clareiras pequenas, houve maior taxa de sobrevivência $(98,90 \%)$ na segunda medição (2006), mas nas medições seguintes a taxa de sobrevivência foi reduzida, certamente, devido ao fechamento do dossel que reduziu a entrada da radiação solar. Nas clareiras grandes, a sobrevivência também foi alta no início do estudo $(97,80 \%)$, e nas duas últimas medições (2008 e 2010), a taxa de sobrevivência não foi alterada $(95,60 \%)$. O fechamento gradual do dossel das clareiras grandes ao longo das medições promoveu um ambiente com uma intensidade de radiação solar que não prejudicou a sobrevivência da espécie.

Outras espécies têm mostrado comportamentos semelhantes ao de Astronium gracile, como, por exemplo, Sterculia pruriens, que, segundo Jardim et al. (2007), possui grande capacidade para adaptar-se em ambientes alterados e Protium paraenses, que apesar de ter caráter tolerante à sombra, como Astronium gracile, pode se adaptar em clareiras, com taxa de mortalidade menor que espécies heliófilas.

Brokaw (1985), ao estudar o comportamento de espécies em ambientes aparentemente desfavoráveis em relação à intensidade luminosa, registrou a ocorrência de 53 espécies tolerantes à sombra regenerando em clareiras de diferentes tamanhos. E Serrão et al. (2003) recomendam ensaios para saber em que nível máximo de radiação as espécies tolerantes à sombra sobrevivem, para responder qual é o nível de abertura suportado por essas espécies, principalmente em atividades que lidam com abertura do dossel.

Barton (1984) afirma que as condições de luminosidade possibilitam a elevação da taxa de crescimento e sobrevivência de espécies tolerantes à sombra em pequenas clareiras. Tanaka e Vieira (2006), ao analisar clareiras de diferentes tamanhos, verificaram que Astronium lecointei, espécie tolerante à sombra, em clareiras de maior extensão, teve melhores resultados fisiológicos. No estudo de Guimarães (2008), Tetragastris altissima, também tolerante à sombra, teve maior sobrevivência no centro das clareiras, onde havia mais luminosidade. Jardim et al. (1995) comentam que espécies tolerantes à sombra são caracterizadas pela capacidade de germinação de suas sementes e desenvolvimento de sua regeneração em condições de sombra, porém, se beneficiam da formação de clareiras.

\section{Crescimento em altura}

O Incremento Periódico Anual em altura de Astronium gracile em cinco anos de monitoramento foi de $36,53 \mathrm{~cm} \cdot a n o^{-1}$, sendo que o maior $\left(43,88 \mathrm{~cm} \cdot \mathrm{ano}^{-1}\right)$ ocorreu na segunda avaliação (2006-2008) e o menor $\left(27,45 \mathrm{~cm}^{-a n o}{ }^{-1}\right)$ ocorreu na terceira avaliação (2008-2010), conforme se pode observar na Figura 2.

Não houve diferença significativa entre os IPAh da primeira, segunda e quarta avaliação, entretanto, o IPAh da terceira avaliação (2008-2010) foi significativamente diferente do IPAh das outras avaliações, de acordo com o teste de Tukey a $5 \%$ de probabilidade.

Nos períodos monitorados, o crescimento em altura das clareiras pequenas foi diferente significativamente das clareiras médias e grandes apenas na segunda avaliação (2006-2008), já as outras avaliações não apresentaram diferença significativa entre os tamanhos de clareira, segundo teste de Tukey a $5 \%$ de probabilidade (Figura 3 ).

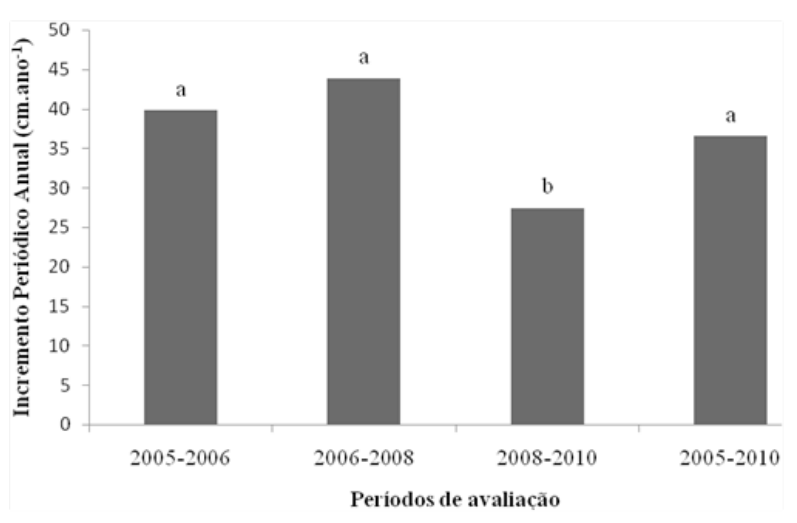

FIGURA 2: Incremento Periódico Anual em altura (IPAh) de mudas de regeneração natural de Astronium gracile Engl., no período de cinco ano, em clareiras causadas por exploração florestal de impacto reduzido, em floresta de terra firme na fazenda Rio Capim, no município de Paragominas, PA. Médias seguidas pela mesma letra não apresentam diferenças estatísticas entre si.

FIGURE 2: Periodic Annual Increment in height of seedlings from natural regeneration of Astronium gracile Engl. in a fiveyear period in different gap sizes in a terra firme natural forest in Rio Capim Forest Management Unit, municipality of Paragominas, Pará state. Bars with a different letter are significantly different at $\mathrm{p}<0.05$. 


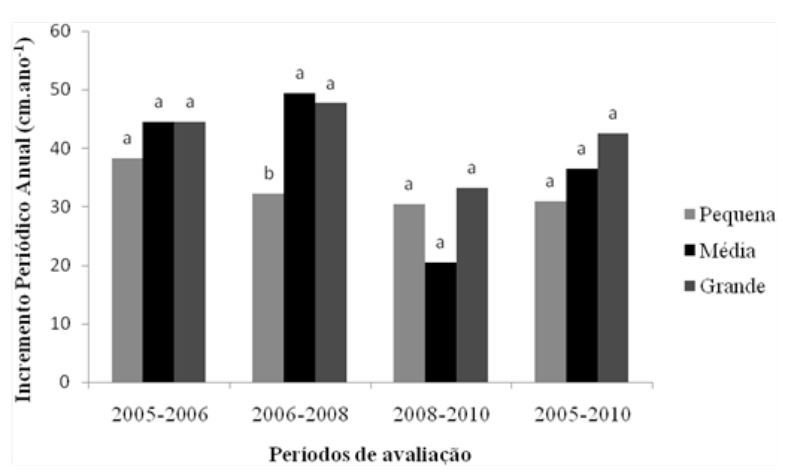

FIGURA 3: Incremento Periódico Anual em altura (IPAh) de mudas de regeneração natural de Astronium gracile Engl., no período de cinco anos, em três tamanhos de clareiras ocasionadas por exploração florestal de impacto reduzido, na fazenda Rio Capim, no município de Paragominas, PA. Médias seguidas pela mesma letra no mesmo período de avaliação não apresentam diferenças estatísticas entre si.

FIGURE 3: Periodic Annual Increment in height of seedlings from natural regeneration of Astronium gracile Engl. in a fiveyear period in three gap sizes in a terra firme natural forest in Rio Capim Forest Management Unit, municipality of Paragominas, Pará state. Bars with a different letter are significantly different at $\mathrm{p}<0.05$.

A maior incidência de radiação solar nas clareiras grandes permitiu que essa espécie tivesse um desempenho melhor em relação às outras clareiras, em todo o período de monitoramento. Isso corrobora o comentário de Costa et al. (2008) de que independente das características intrínsecas das espécies (grupo ecológico, fatores genéticos etc.), a entrada de luz na copa das árvores estimula um bom crescimento de todas as espécies. Poggiani et al. (1992) também afirmam que o incremento em altura representa um dos aspectos do crescimento geral de espécies arbóreas, sendo influenciado de forma acentuada pelas condições de luminosidade.

De acordo com Scalon e Alvarenga (1993), espécies que se comportam bem em intensidade luminosa reduzida e também sob céu aberto são espécies indicadas para sistema de enriquecimento. No presente estudo, Astronium gracile teve alta taxa de sobrevivência em todo o período monitorado, mas, principalmente, quando as clareiras ainda estavam mais abertas, no início do processo de fechamento do dossel (Figura 1). Seu incremento em altura também foi maior nas clareiras grandes, também no início do processo de fechamento do dossel (Figuras 2 e 3). Portanto, a espécie pode ser indicada para compor a regeneração natural que poderá formar estoques de madeira para o futuro.

\section{CONCLUSÕES}

As mudas de regeneração natural de Astronium gracile Engl. beneficiaram-se com a abertura do dossel da floresta, causada pela exploração florestal, principalmente nas clareiras médias $\left(401-600 \mathrm{~m}^{2}\right)$ e grandes (área $\left.>600 \mathrm{~m}^{2}\right)$, onde cresceram mais em altura e tiveram maior taxa de sobrevivência, demonstrando potencial para uso em sistemas silviculturais, para aumentar o valor comercial da floresta para futuras colheitas de madeira.

\section{REFERÊNCIAS BIBLIOGRÁFICAS}

BAZZAZ, F. A.; PICKETT, S. T.A. Physiological ecology of tropical succession: a comparative rewiew. Annual Review of Ecology and Systematics, New York, v. 11, p. 287-310, Nov. 1980.

BARTON, A. M. Neotropical pioneer and shadetolerant tree species: do the partition trefall gaps. Tropical Ecology, New York, v. 25, p. 196-202, 1984.

BROKAW, N. V. L. Gap-phase regeneration in a tropical forest. Ecology, Durham, v. 66, n. 3, p. 682687, 1985.

CARON, B. O. et al. Crescimento em viveiro de mudas de Schizolobium parahyba (Vell.) S. F. Blake. Submetidas a níveis de sombreamento. Ciência florestal, Santa Maria, v. 20, n. 4, p. 683689, out./dez. 2010.

CARVALHO, J. O. P. Classificação em grupos ecológicos das espécies mais importantes em uma área de floresta Nacional do Tapajós. Belterra, PA. Belém: Embrapa Amazônia Oriental, 2000. 4 p. (Embrapa Amazônia Oriental. Comunicado Técnico, 41).

COSTA, D. H. M.; SILVA, J. N. M.; CARVALHO, J. O. P. de. Crescimento de árvores em uma área de terra firme na floresta nacional do tapajós após a colheita de madeira. Revista de Ciências Agrárias, Belém, n. 50, p. 63-76, jul./dez. 2008. 
DENSLOW, J. S. Gap partitioning among tropical rain forest trees. Biotropica, Austrália, v. 12, p. 4755,1980 .

GOMES, J. M. et al. Sobrevivência de espécies arbóreas plantadas em clareiras causadas pela colheita de madeira em uma floresta de terra firme no município de Paragominas na Amazônia brasileira. Acta Amazonica, Manaus, v. 40, n. 1, p. 171-178, 2010.

GUIMARÃES, R. de P. M. Avaliação da regeneração natural de Tetragastris altissima (Aubl.) Swart em clareiras abertas em florestas exploradas seletivamente. 2008. 100 f. Dissertação (Mestrado em Botânica Tropical) - Universidade Federal Rural da Amazônia/ Museu Paraense Emílio Goeldi, Belém, 2008.

JARDIM, F. C. da S. et al. Dinâmica da vegetação arbórea com DAP menor que 5,0 $\mathrm{cm}$ na Estação Experimental de Silvicultura Tropical do INPA, Manaus - AM. Boletim da FCAP, Belém, v. 23, p. 7-32, 1995.

JARDIM, F. C. da S.; SERRÃO, D. R.; NEMER, T. C. Efeito de diferentes tamanhos de clareiras, sobre o crescimento e a mortalidade de espécies arbóreas, em Moju-PA. Acta Amazonica, Manaus, v. 37, n.1, p. 37-48, 2007.

JARDIM, F. C. da S.; SOARES, M. da S. Comportamento de Sterculia pruriens (Aubl.) Schum. em floresta tropical manejada em MojuPA. Acta Amazonica, Manaus, v. 40, n. 3, p. 535542, 2010.

HARTSHORN, G. S. Neotropical forest dynamics. Biotropica, Austrália, v. 12, p. 23-30, 1980.

MARTINS, S. V. et al. Caracterização do dossel e do estrato de regeneração natural no sub-bosque e em clareiras de uma floresta estacional semidecidual no município de Viçosa-Mg. Revista Árvore, v. 32, n. 4, p. 759-767, 2008.

OLIVEIRA, L. C. de. et al. Exploração florestal e eficiência dos tratamentos silviculturais realizados em uma área de 136 ha na Floresta Nacional do Tapajós, Belterra-Pará. Revista de Ciências Agrárias, Belém, n. 46, p. 195-213, 2006.

ORIANS, G. H. The influence of tree-falls in tropical forests in tree species richness. Tropical Ecology, New York, v. 23, n. 2, p. 255-279, 1982.

PINTO, A. M.; VARELA, V. P.; BATALHA, L. F. P. Influência do sombreamento no desenvolvimento de mudas de Louro pirarucu (Licaria canella (Meissn.) Kosterm). Acta Amazonica, Manaus, v.
23, n. 4, p. 397-402 1993.

POGGIANI, F.; BRUNI, S.; BARBOSA, E. S. Q. Efeito do sombreamento sobre o crescimento das mudas de três espécies florestais. Revista do Instituto Florestal de São Paulo, v. 4, n. 2, p. 564$569,1992$.

RODRIGUES, T. E. et al. Caracterização e classificação dos solos do município de Paragominas, Estado do Pará. Belém: Embrapa Amazônia Oriental, 2003. 51 p. (Documentos 162). RONDON NETO, R. M. et al. Estrutura e composição florística da comunidade arbustivoarbórea de uma clareira de origem antrópica, em uma floresta estacional semidecídua montana, Lavras-MG, Brasil. Cerne, Lavras, v. 6, n. 2, p. 079-094, 2000.

SANSEVERO, J. B. B.; PIRES, J. P. de A.; PEZZOPANE, J. E. M. Caracterização ambiental e enriquecimento da vegetação de áreas em diferentes estágios sucessionais (pasto, borda, clareira e floresta). Revista Científica Eletrônica de Engenharia Florestal, Garça, v.6, n. 7, fev. 2006.

SCALON, S. P. Q.; ALVARENGA, A. A. Efeito do sombreamento sobre a formação de mudas de Pau-pereira (Platycyamus regnelli Benth). Revista Árvore, v. 17, n. 3, p. 265-270, 1993.

SERRÃO, D. R.; JARDIM, F. C. da S.; NEMER, T. C. Sobrevivência de seis espécies florestais em uma área explorada seletivamente no município de Moju, Pará. Cerne, Lavras, v. 9, n. 2, p. 153-163, jul./dez. 2003.

SWAINE, D.; WHITMORE, T. C. On the definition of ecological species groups in tropical rain forests. Vegetatio, v. 75, p. 81-86, 1988.

TANAKA, A.; VIEIRA, G. Autoecologia das espécies florestais em regime de plantio de enriquecimento em linha na floresta primária da Amazônia Central. Acta Amazonica, Manaus, v. 36, n. 2, p. 193-204, 2006.

VELOSO, H. P.; RANGEL FILHO, A. L. R.; LIMA, J. C. A. Classificação da vegetação brasileira adaptada a um sistema universal. IBGE, Departamento de Recursos Naturais e Estudos Ambientais, Rio de Janeiro, 1991, 124 p.

WATRIN, O. S.; ROCHA, A. M. A. Levantamento de vegetação natural e uso da terra no Município de Paragominas (PA) utilizando imagens TM/ Landsat. Belém: EMBRAPA-CPATU, 1992, 40 p. (Boletim de Pesquisa, 124). 